\title{
A Systematic Review of Effective Modifications to Cognitive Behavioural Therapy for Young People with Autism Spectrum Disorders
}

\author{
Sasha Walters $^{1,2} \cdot$ Maria Loades $^{1} \cdot$ Ailsa Russell $^{1}$
}

Received: 22 October 2015 / Accepted: 11 February 2016/Published online: 21 March 2016

(C) The Author(s) 2016. This article is published with open access at Springerlink.com

\begin{abstract}
The present review evaluated studies which effectively employed cognitive behavioural therapy (CBT) to alleviate symptoms of common mental health problems in young people with autism spectrum disorder (ASD). It assessed the modifications applied to CBT and compliance with recent guidelines from the National Institute of Health Care Excellence (NICE). Systematic searches of electronic databases, reference lists, and journals identified 12 studies meeting predetermined inclusion criteria. Results indicated that modified CBT yielded reductions in anxiety, obsessivecompulsive disorder (OCD), and depression. There was a lack of gold standard research into the effects of CBT for disorders other than anxiety. A greater number of modifications than recommended by NICE were consistently employed, including disorder-specific modifications. Implications for clinical intervention and future research are discussed.
\end{abstract}

Keywords Autism spectrum disorders - Cognitive behaviour therapy $(\mathrm{CBT}) \cdot$ Anxiety $\cdot$ OCD $\cdot$ Depression $\cdot$ Young people

The prevalence of autism spectrum disorder (ASD) ranges from 0.6 to $1.0 \%$ of the child and adolescent population (Gillberg and Billstedt 2000; Simonoff et al. 2008). Increasing numbers of people are diagnosed with ASD and comorbid mental health problems in the UK, placing increased demands on services (Langdon et al. 2013). Among

Sasha Walters

slwalters85@gmail.com

1 University of Bath, Claverton Down, Bath BA2 7AY, UK

2 Now at Child and Adolescent OCD Team, Michael Rutter Centre, South London and Maudsley NHS Foundation Trust, London, UK young people ( $\leq 18$ years of age) with ASD, mood and affective disorders have been reported to occur at a greatly increased rate when compared to rates among typically developing populations (Ozsivadjian and Knott 2011; Whitehouse et al. 2009). Mental health comorbidities can result in more frequent referrals into services (Matson and Nebel-Schwalm 2007), and one in ten young people engaging with Child and Adolescent Mental Health Services (CAMHS) is thought to have an ASD (Wistow and Barnes 2009). The additional impairment for young people and increased burden on families and mental health services has increased the necessity for successful and cost-effective treatments for comorbid mental health needs in the context of ASD (e.g. Donoghue et al. 2011; Kannabiran and McCarthy 2009; Langdon et al. 2013; Reichow et al. 2011).

Cognitive behaviour therapy (CBT) has been proposed as a potential intervention for young people with ASD. There is a substantial evidence base relating to the effectiveness of CBT in treating mood and affective disorders in typically developing young people (e.g. Cartwright-Hatton et al. 2004; POTS 2004; Reinecke et al. 1998; Wethington et al. 2008). However, core features of ASD such as social communication difficulties, concrete thinking and deficits in emotional literacy (Baron-Cohen et al. 1985; Leyfer et al. 2006; Minshew et al. 1997; Ozonoff et al. 1991; Simonoff et al. 2008) are thought to inhibit the efficacy of standard treatment (Lickel et al. 2012). This has led to a debate about whether CBT is appropriate for this population (Chalfant et al. 2007; Lickel et al. 2012).

There have been a number of narrative reviews (Donoghue et al. 2011; Reaven et al. 2009; Rotheram-Fuller and MacMullen 2011; Scattone and Mong 2013; White et al. 2009) and one systematic review (Lang et al. 2010) which have collated the findings of empirical studies evaluating the effectiveness of CBT for people with ASD. The majority of these reviews include four to nine studies with designs ranging 
from randomised controlled trials to uncontrolled treatment evaluations. Most reviews are focused on studies considering the use of CBT in treating anxiety in young people with ASD. Conclusions are largely in favour of CBT as an effective intervention. Three of the six reviews conducted identify modifications to CBT in order to enhance efficacy for young people with ASD (Donoghue et al. 2011; Reaven et al. 2009; Rotheram-Fuller and MacMullen 2011). However, the reviews often lack a clear critique of the quality of study design and the lack of systematic reviews limits the extent to which conclusions can be drawn regarding the effective application of modifications to clinical interventions.

Despite this, such studies have informed the guidance recently published by NICE to inform clinical management and support of children and young people on the autism spectrum (Baird et al. 2013; Guideline Development Group). This document recommends a number of modifications when using CBT for anxiety in young people with ASD as detailed in Table 1.

As shown in Table 1, the modifications are largely focused on the structure and mode of delivery of CBT rather than the content of the intervention and point to the need to reduce, or simplify, cognitive components. The guideline acknowledges additional mental health problems including depression, obsessive-compulsive disorder (OCD), body dysmorphic disorder (BDD) and post-traumatic stress disorder (PTSD). However, rather than referring to disorder-specific modifications for any of these disorders, clinicians are advised to follow recommendations for typically developing young people.

It is believed that the lack of specific guidance in relation to disorders other than anxiety may reflect the limited or weak published evidence (Wood et al. 2011). While young people with ASD 'may be candidates for talk-based therapies similar to those employed with children and adults with mental health disorders' (Wood et al. 2011, p. 197), this has yet to be consistently empirically confirmed. It is important to ensure that clear and comprehensive guidelines pertaining to the delivery

Table 1 NICE guidance (CG170 2013, p. 22) regarding modifications to CBT for ASD and anxiety

NICE recommended modifications to apply to CBT for young people with ASD and anxiety

1. Emotion recognition training

2. Greater use of written and visual information and structured worksheets

3. A more cognitively concrete and structured approach

4. Simplified cognitive activities, for example, multiple choice worksheets

5. Involving a parent or carer to support the implementation of the intervention, for example, involving them in therapy sessions

6. Maintaining attention by offering regular breaks

7. Incorporating the child or young person's special interests into therapy if possible of effective interventions are available to support consistency in the administration of successful treatment for the broad spectrum of comorbid mental health disorders in young people with ASD (Wood et al. 2011). Specifically, there has been a call to "determine the core ingredients of effective treatment, how traditional CBT strategies may need to be modified for children with ASD, and how treatment should be delivered' (White et al. 2009, p. 18).

This paper seeks to respond to this call and provide a comprehensive review of published original studies using CBT to treat mood and affective disorders in young people with ASD. It seeks to build on existing systematic reviews (e.g. Lang et al. 2010; Scattone and Mong 2013; Vasa et al. 2014; White et al. 2009; Wood et al. 2011) by critically appraising the quality, efficacy and nature of modifications to CBT reported in the treatment of anxiety as well as OCD and depression, in young people with ASD. Crucially, this review aims to adopt a systematic search and review of the literature in order to draw robust conclusions about how CBT should be modified to effectively reduce symptoms of comorbid mental health disorders in young people with ASDs. The specific research questions being asked of this literature include:

1. How many published studies report a significant effect of a CBT intervention, for young people with ASD and comorbid anxiety, OCD or depression?

2. Are these interventions using the modifications recommended by NICE guidance?

3. Are additional adaptations being employed that have implications for practice?

The objective of considering these questions is to provide a comprehensive document which can be used to supplement NICE CG170 recommendations and inform clinical practice with a typically hard to reach population who could benefit from therapeutic interventions but may struggle to access standardised approaches (Langdon et al. 2013; Wood et al. 2011).

\section{Method}

A systematic review was conducted according to the Preferred Reporting Items for Systematic Reviews and Meta Analyses (PRISMA; Liberati et al. 2009) to improve the rigour of data extraction and reporting.

Protocol Methods of review and inclusion criteria were specified in a research proposal that was reviewed for feasibility, a priori, by the second and third authors. All authors are qualified clinical psychologists with experience of cognitive behavioural interventions with young people with ASD and comorbid mental health problems as well as having experience of evaluating research in the fields of CBT and ASD. 
Eligibility Criteria: Inclusion Criteria Studies reporting original outcome data about a CBT intervention for young people with ASD and comorbid mental health problems were included. Inclusion criteria followed the PICOS approach recommended in PRISMA (Liberati et al. 2009) to identify participants, interventions, comparators, outcomes and study design of interest.

Types of Participants Children and young people ( $\leq 18$ years of age) with a diagnosis of ASD (autism, Asperger's or pervasive developmental disorder-not otherwise specified (PDDNOS)). Diagnosis of ASD had to be confirmed within the study design section, and methods include a standardised assessment tool such as the Autism Diagnostic Observation Schedule (ADOS) (Lord et al. 1989). Participants also had to have scores within the clinical range on standardised measures of mental health symptoms such as anxiety, OCD or depression.

Intervention Studies were included if they reported using a CBT intervention to ameliorate mental health symptoms. The method sections were screened to ensure that studies had (a) employed a manualised CBT intervention or (b) reported cognitive and behavioural intervention techniques in keeping with those described by Velting et al. (2004) as necessary components of a CBT intervention. Absence of reported modifications to the CBT intervention was not an exclusion criterion as efficacy of non-modified CBT for this population would have been of equal interest. However, all eligible studies reported some degree of modification.

Studies reporting on interventions for OCD were reviewed separately to studies employing an intervention for anxiety despite the fact that many anxiety studies included participants with a diagnosis of OCD. The anxiety studies did not report on the efficacy of the intervention by diagnosis, and treatment protocols have been developed for treating OCD in children which are distinct from anxiety treatments (e.g. March and Mulle 1998). It was considered clinically relevant to review the effects of these interventions separately.

Comparator The treatment group had to be compared to a control population, who either received an alternative intervention or were waitlisted for the duration of the study. Single case design studies and studies that did not have a comparator group were excluded as the primary focus of this study was on effective interventions, and it is difficult to infer efficacy of a specific intervention with no comparison group.

Outcome The primary outcome of interest for the current study was the modifications applied to an effective CBT intervention. For the purposes of this review, 'efficacy' was defined as (a) a statistically significant reduction in target mental health symptoms from pre- to post-treatment and/or (b) a clinically meaningful change in symptoms such that post-treatment scores were below the clinical cutoff of a scale or criteria for diagnosis was no longer met.

Study Design Randomised control trials (RCTs) and casecontrol studies were included provided the above criteria were met. Studies had to include measures of mental health symptoms, and symptoms must have been measured at pre- and post-intervention as a minimum.

Exclusion Criteria Non-English language studies were excluded due to lack of resources for translation. The decision was also made to exclude all grey material for two main reasons: there is a risk of bias through including literature which has not successfully passed peer review where methodology has the potential to be less rigorous. Furthermore, in order to address the question posed by this review, it was necessary to consider studies with significant effects and studies which do not yield clinically significant effects typically do not achieve publication (Hopewell et al. 2007).

Information Sources and Search Terms Systematic searches of four electronic databases were included: PubMed, Scopus, PsychINFO and WEB of SCIENCE. Publication year was not limited. Reference lists of mostcited articles and recent review papers were searched by hand, as were databases of the journals most frequently used (Journal of Autism and Developmental Disorders, Journal of Child Psychology and Psychiatry).

The following terms were used: 'CBT' or 'Cognitive Behaviour Therapy', ‘Autism', 'Young People' (also children and adolescents separately) and '[mental health disorder] (anxiety, depression, OCD, BDD, PTSD; no papers were found for the BDD search in any search engines and only one case study was found for PTSD so these disorders are not referred to within results). Searches were initially expanded to include specific mental health disorders and young people. This was followed by a simplified search including just 'CBT' and 'Autism' ((CBT [Title/Abstract] AND autism [Title/Abstract]) (PubMed example) which returned all studies identified in the more complex search plus additional relevant studies. Overall search results are reported in the PRISMA flow diagram (see Fig. 1).

Study Selection and Data Extraction Process The first author completed the searches and reviewed the title and abstract of all returned results to confirm whether studies met eligibility criteria. Of those studies which met eligibility criteria, the first author completed data extraction on all data items of interest for the research question including participants, intervention characteristics, study design and measures, efficacy of intervention at reducing mental health symptoms (pre- and post-measures, statistical significance and report of change 
index or results in relation to clinical cutoff) and modifications to interventions. The second and third authors reviewed the data extraction table to confirm study inclusion and although frequent consultation was had between authors on study selection and data extraction, the second and third authors did not complete independent inter-ratings of theses stages.

Risk of Bias The Newcastle-Ottawa Scale of assessment (NOS) was employed as a quality assessment tool. The NOS is recommended by the Cochrane Handbook as suitable for the evaluation of non-randomised clinical trials and thus able to assess quality across the range of study designs captured by the present review. The NOS permitted assessment of risk of bias in individual studies across participant selection (score range 0-4), comparability of treatment to control group (score of 0-2) and measure of exposure (impact) of treatment (score $0-3)$. Overall scores were categorised into high (1-3), moderate (4-6) and low (7-9) risk of bias. An additional scale was developed for the purpose of this review to assess the content of CBT within the modified intervention. This scale followed the structure of the NOS scales and was designed to measure adherence to the six components of CBT as identified by Velting et al. (2004). These comprise psychoeducation, somatic management, cognitive restructuring, problem solving, exposure and relapse prevention. Full adherence to the CBT model or clearly defined cognitive and behavioural components was summarised to provide a score (0-1). A score of 1 denotes either (a) evidence of all six components of CBT or (b) clear evidence of core cognitive and behavioural components (e.g. cognitive restructuring and exposure).

\section{Results}

Study Selection Titles and abstracts of the 468 studies initially identified were scanned according to the eligibility criteria. Non-eligible studies and duplicate titles were removed. This resulted in 39 full text articles being considered for review. Data was extracted from each study and was summarised in terms of (a) participant characteristics, (b) quality of study design and measures according to the NOS, (c) efficacy of intervention at reducing mental health symptoms and (d) modifications to interventions including the extent to which cognitive components of intervention were retained. At this stage, a further 27 studies were excluded from the final review of data for the following reasons. Eight studies were reports of secondary data (Boyd et al. 2012; King and Desaulnier 2011; Lang et al. 2010; Langdon et al. 2013; Reaven 2009, 2011, Reaven et al. 2009; Rotheram-Fuller and MacMullen 2011; Scattone and Mong 2013), one study (Sze and Wood 2008)
Fig. 1 PRISMA flow diagram of study inclusion. From: Moher et al. (2009)

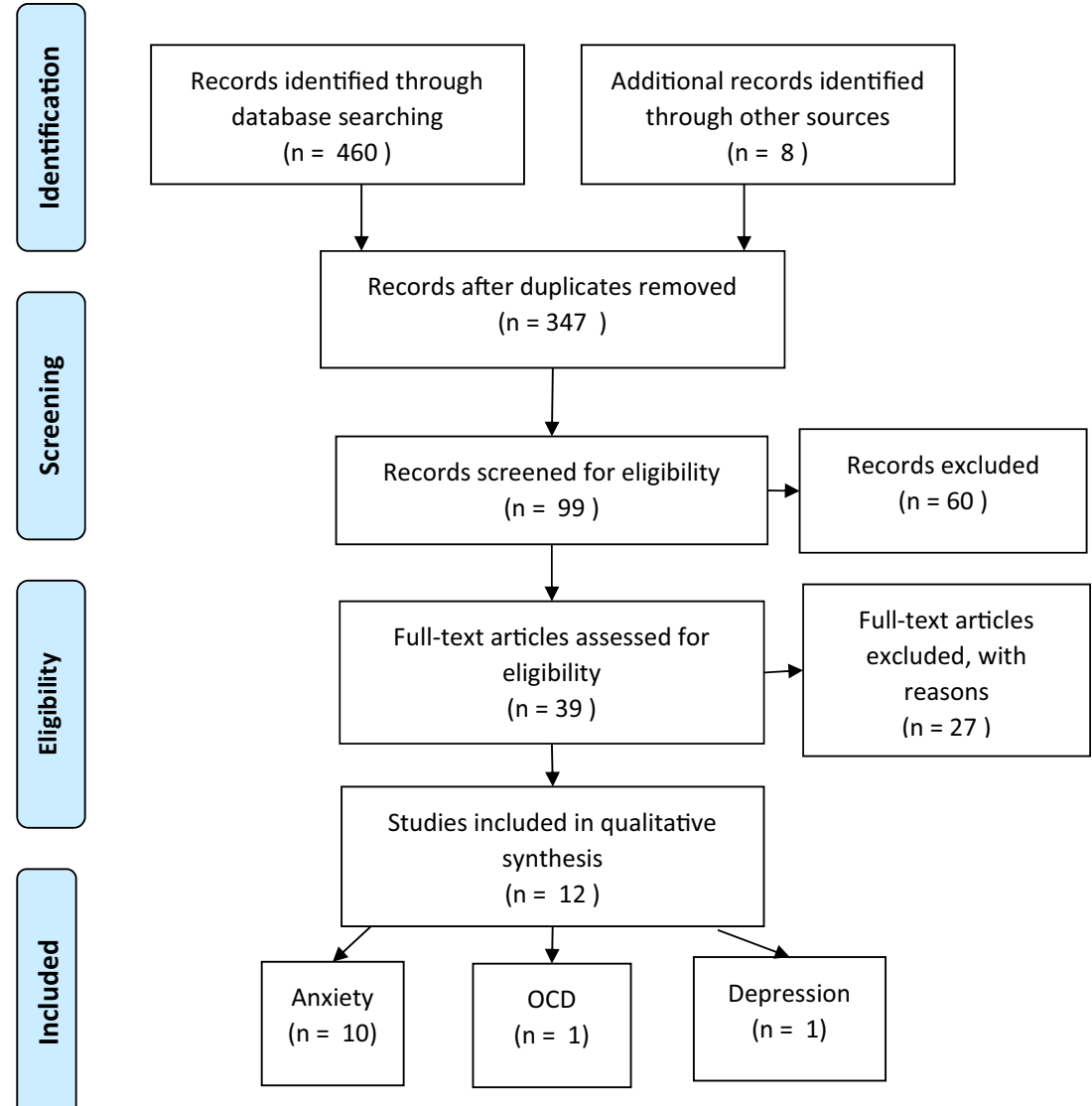


was a duplicate that had not been previously filtered out, one (White et al. 2013) reported a non-significant effect of the intervention, five studies reported the effects of CBT for core features of ASD rather than mental health symptoms (Drahota et al. 2011; Kenworthy et al. 2014; Scarpa and Reyes 2011; Wood et al. 2009a, b, 2014) and 12 included $(n=1)$ designs (Cook et al. 1993; Lehmkuhl et al. 2008; Nadeau et al. 2014; Reaven and Hepburn 2003; Schleismann and Gillis 2011; Sze and Wood 2007, 2008) or did not have a comparator group (Reaven et al. 2012b; Ooi et al. 2008; Ozsivadjian and Knott 2011; White et al. 2010; White et al. 2009).

\section{Outcome of Interventions}

\section{Anxiety Disorders}

The current study reviewed 10 studies which met eligibility criteria to answer the primary research questions. Results follow subheadings from the NOS scale to summarise study characteristics and expand upon scores detailed in Table 2 relating to risk of bias in interpretation of findings.

Participants A total of 423 young people with ASD and comorbid anxiety were recruited to group or individual CBT-based interventions with sample sizes ranging from 12 to 71. Study designs included controlled trials and RCTs (Chalfant et al. 2007; Fuji et al. 2013; McNally Keehn et al. 2013; Reaven et al. 2012a; Reaven et al. 2009; Sofronoff et al. 2005; Storch et al. 2013; Sung et al. 2011; Wood et al. 2009a; Wood et al. 2015).

The majority of participants were male $(n=353$ or $83.5 \%$; 70 females), which broadly equates to the ratio of males to females diagnosed with ASD (4:1; Baron-Cohen et al. 2001). Ages ranged from 7 to 16 years with the majority of studies recruiting older children and just one study recruiting adolescents (Wood et al. 2015). All participants were high functioning with average or above IQ. The majority of participants had a diagnosis of high functioning autism (HFA; $47.7 \%$ ) or Asperger's syndrome (28.4\%) with the remainder $(10.2 \%)$ described as having PDDNOS or jointly categorised as autism with PDD-NOS $(13.7 \%)$. The spectrum of anxiety disorders was identified and treated including social phobia, separation anxiety, specific phobias, generalised anxiety disorder, panic disorder, agoraphobia and OCD.

\section{Participant Selection and Comparability to Controls}

The majority of studies included strong participant selection methods with eight of the studies achieving a score of 3-4/4. The two remaining studies (McNally Keehn et al. 2013; Sofronoff et al. 2005) scored 2/4 due to potential selection bias limiting the representativeness of their samples. Sofronoff et al. (2005) recruited through community adverts rather than clinics, potentially introducing bias by including participants who self-refer to studies. McNally Keehn et al. (2013) described recruitment through local agencies and nonprofit organisations, but there was not sufficient detail to permit replication. Studies were typically poor on defining whether the anxiety problem was of recent onset or a historical difficulty. In terms of concomitant medication, $50 \%$ of the studies indicated that participants were accepted if they were medicated providing the dose was stable (Fuji et al. 2013; Reaven et al. 2009; Storch et al. 2013; Sung et al. 2011; Wood et al. 2009a, b). There were strengths in respect of the validation of case definition with all studies confirming ASD diagnosis via a standardised measure such as the ADOS (Lord et al. 1989). The majority also confirmed the diagnosis of anxiety with an interview such as the Anxiety Disorders Schedule for Children/Parents (ADIS-C/P; Silverman and Albano 1996), although one (Sung et al. 2011) relied on the child Spence Children's Anxiety Scale (SCAS; Nuata et al. 2004). All studies reported random allocation to the treatment or comparator condition, but just $50 \%$ of the studies actively assessed comparability of participants to controls either through matching based on demographics in the study design or controlling for baseline anxiety in the analysis (Fuji et al. 2013; McNally Keehn et al. 2013; Sofronoff et al. 2005; Wood et al. 2009a, b, 2015).

Intervention Characteristics The duration of interventions ranged from 6 to 32 sessions (modal number 16 sessions) lasting between 50 and $120 \mathrm{~min}$ (modal time $90 \mathrm{~min}$ ). Four studies delivered a group intervention (Chalfant et al. 2007; Reaven et al. 2012a; Sofronoff et al. 2005; Sung et al. 2011), one study delivered a group intervention with individual components (Reaven et al. 2009) and the remaining five studies evaluated an individual intervention. Studies employed a variety of designs including intervention compared to waitlist (WL; $n=5$ ), intervention compared to treatment as usual (TAU; $n=3$ ), child compared to child plus parent compared to WL $(n=1)$ and CBT intervention compared to a social programme $(n=1)$.

Five studies modified CBT programmes designed for typically developing young people including Building Confidence (Wood and McLeod 2008), Coping Cat (Kendall 1992), Cool Kids (Lyneham et al. 2003) and Exploring Feelings (Attwood 2004). Three studies employed manuals specifically developed for young people with autism (Facing your Fears; Reaven et al. 2009; Reaven et al. 2012a) or an unstandardized programme (Sofronoff et al. 2005); two studies (Storch et al. 2013; Wood et al. 2015) employed Behavioural Interventions for Anxiety in Children with Autism (BIACA; Wood and Drahota 2005). 












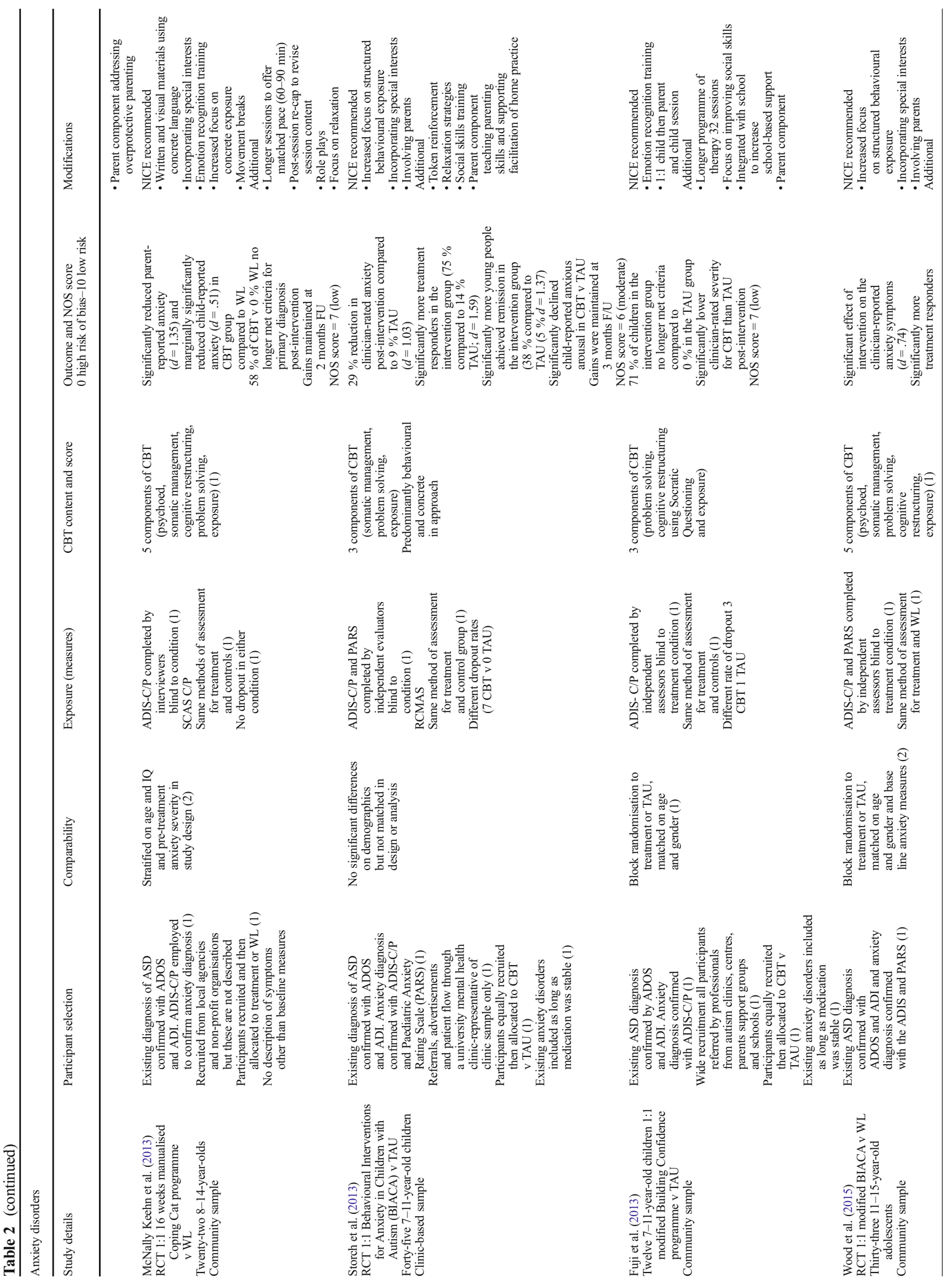




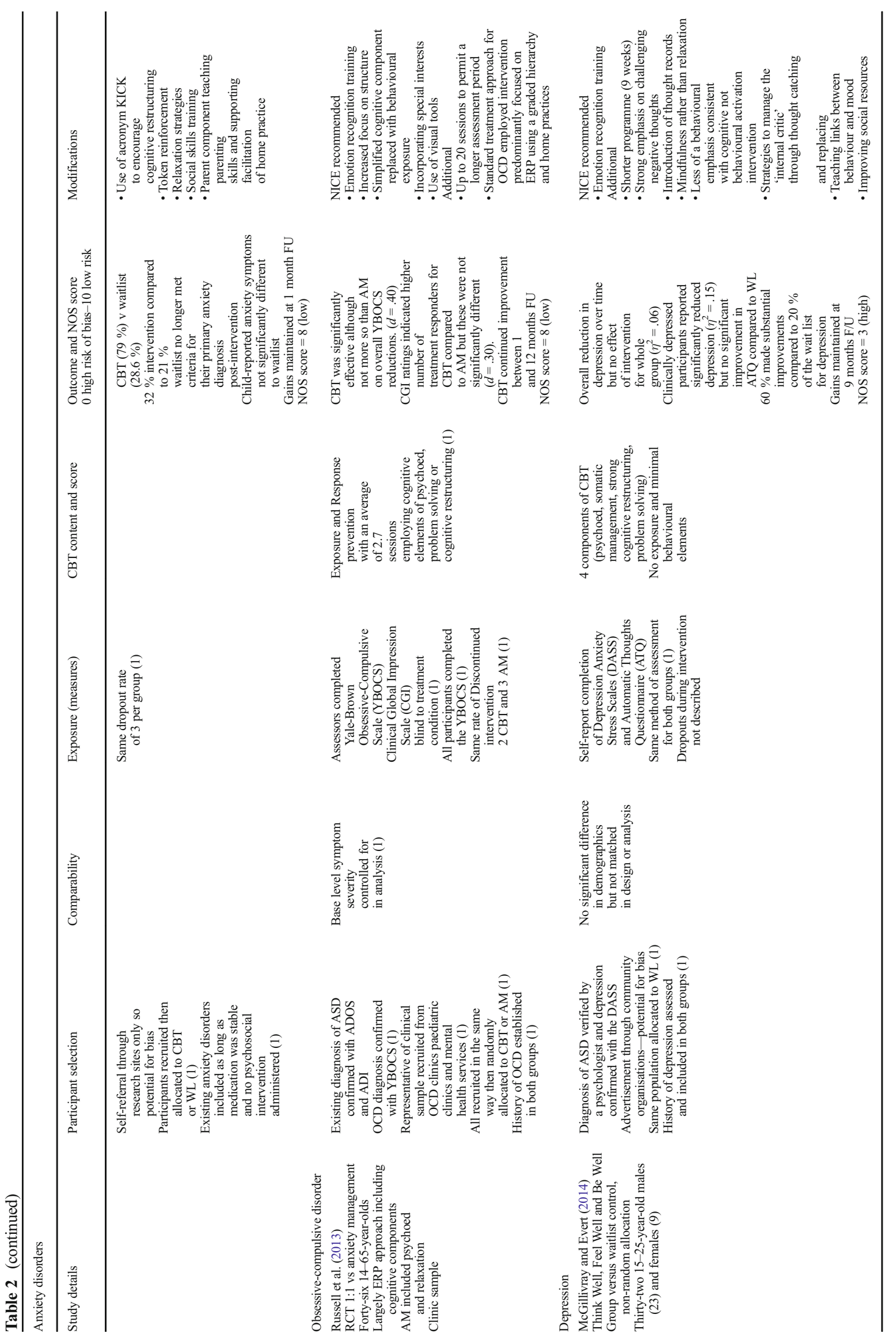


CBT Component All studies described between three and six components of CBT. The most frequently reported were psychoeducation about emotions particularly affect recognition, problem solving and exposure to feared outcomes. The majority of studies reported a reduced cognitive component with greater employment of behavioural strategies such as exposure and relaxation. Relaxation activities were delivered in a more directive way than would be expected for CBT with a typically developing population. Cognitive restructuring was typically delivered in a creative way through the use of acronyms such as KICK-Knowing I'm nervous, Icky thoughts, Calming thoughts, Keep practicing (Wood et al. 2015); through guided discovery pretending to be scientists (Sofronoff et al. 2005); or through the use of lists of unhelpful and helpful thoughts from which alternative thinking strategies could be chosen rather than generated. Similarly, problem solving was introduced through acronyms such as STARStop, Think, Act, Reflect (Sung et al. 2011) —or social stories and most exposure was completed as home practices. Relapse prevention plans were not reported, with the exception of two studies (Chalfant et al. 2007; Sofronoff et al. 2005).

It is of interest to note that it was the studies employing five or six components of CBT (Chalfant et al. 2007; McNally Keehn et al. 2013; Sofronoff et al. 2005; Sung et al. 2011; Wood et al. 2015) which found significant reductions in child reports of anxiety or an increased use of coping strategies.

\section{Ascertainment of Exposure (Outcome Measures) A variety} of measures were used across the studies to assess change in anxiety symptoms. All studies relied on standardised measures validated in a typically developing population, rather than with samples of young people with ASD. The most commonly used measures included an interview (ADIS-C/P) and a parent and child report questionnaire (SCAS). Sofronoff et al. (2005) used an idiographic measure to assess change in the ability to generate strategies to manage anxiety which was developed specifically for young people with ASDs. All studies employed the same measures across control and treatment groups demonstrating a strength of ascertainment of impact. More than half the studies employed a multi-informant design and incorporated a mix of questionnaire/rating scales and interviews, reporting on parent and/or child report, as well as clinician-based observation ratings (Chalfant et al. 2007; McNally Keehn et al. 2013; Storch et al. 2013; Sung et al. 2011; Wood et al. 2009a, b, 2015). Six studies also reported rigour in methods in this area, reducing bias by including independent evaluators, blind to treatment condition, to complete measures of anxiety (Fuji et al. 2013; McNally Keehn et al. 2013; Reaven et al. 2012a; Storch et al. 2013; Wood et al. 2009a, b, 2015). Bias was introduced to studies through variation in reports of non-response across studies, including no dropout in either group (McNally Keehn et al. 2013; Sofronoff et al. 2005), equal rates (Sung et al. 2011; Wood et al. 2009a, b, 2015), different rates across groups (Fuji et al. 2013; Storch et al. 2013) and dropout not being reported for the control group (Chalfant et al. 2007; Reaven et al. 2009, 2012a, b).

Outcomes and Overall Risk of Bias As a requirement of the review, all studies reported a positive effect of intervention at reducing anxiety on at least one measure. One study demonstrated a significant effect of the intervention, but this was not significantly different to the control intervention (Social Recreation Program; Sung et al. 2011). All studies reported pre- and post-treatment effects, in addition to at least one follow-up measure indicating that gains had been maintained over time, with the exception of three studies (Chalfant et al. 2007; Fuji et al. 2013; Reaven et al. 2009).

Four studies found child-reported reductions in anxiety (Chalfant et al. 2007; McNally Keehn et al. 2013; Sung et al. 2011; Wood et al. 2015), one study found childreported reduction in anxious arousal (Storch et al. 2013) and one reported that children demonstrated an increased use of strategies to cope with anxiety (Sofronoff et al. 2005). All 10 studies reported a parent- and/or clinician-rated reduction in anxiety. However, only $6 / 10$ used clinician ratings blind to treatment condition and as all parents were involved in the treatment process (with the exception of McNally Keehn et al. (2013)), possible bias arising from investment in outcome may exist. Where effect sizes were reported, all were classified as 'large' with the exception of Sung et al. (2011). These authors reported between group differences in child-reported anxiety over time with a small effect size of .06. A small effect size is consistent with the lack of significant difference found between the CBT and comparator intervention.

The effective studies included a mix of individual (Fuji et al. 2013; McNally Keehn et al. 2013; Storch et al. 2013; Wood et al. 2009a, b, 2015) and group (Chalfant et al. 2007; Reaven et al. 2009, 2012a; Sofronoff et al. 2005; Sung et al. 2011) interventions. The majority of studies achieved scores of between 4 and 6 on the NOS indicating a moderate risk of bias. Four studies achieved a score of 7 or 8 indicating low risk of bias (Fuji et al. 2013; McNally Keehn et al. 2013; Wood et al. 2009a, b, 2015), but no study achieved a full score on this scale. Typical areas of weakness across studies included a lack of reported history of symptoms across the treatment and control group, a lack of independent evaluators of outcome, blind to treatment condition and narrow recruitment from nonclinical populations. Each of these factors introduce the potential for bias within the sample or interpretation of effect.

Modifications Only two studies employed all seven of the NICE recommended modifications (Reaven et al. 2009, 2012a), and these developed a tailored treatment manual for the study rather than using a modified version of an existing manual. All studies implemented the NICE recommendations 
Table 3 Summary of recommended modifications to the content and delivery of interventions for anxiety

Recommended adaptations to CBT for anxiety disorders

- Longer duration of sessions to allow more time to match children's pace and repeat content to aid learning

- Use of metaphors, e.g. child as scientist to encourage guided discovery

- Use of acronyms, e.g. STAR and KICK to introduce problem solving and cognitive restructuring

- Use of social stories for cognitive restructuring and problem solving (e.g. antidote to noxious thoughts; Sofronoff et al. 2005)

- Use of idiosyncratic rating scales such as James and the Maths test and a feelings thermometer to concretely measure change instead of asking about feelings directly

- Incorporate a relaxation strategy section into the programme to support affect management concretely

- Tangible reinforcement programme in session which can be translated to home and school such as a token reinforcement programme

- Use of video modelling and role play to teach coping strategies

- Increased use of games to convey concepts and maintain interest for younger children

- Employ an additional parenting component to teach parents about the role of overprotective parenting in anxiety disorders and strategies to support their child and manage their own feelings of anxiety

- Link with schools to increase school-based support and generalisation of concepts

regarding the use of visual aids and providing emotion recognition. All apart from one study (McNally Keehn et al. 2013) involved parents, either as co-therapists in sessions or through a separate parent component. There were also a wide range of additional modifications employed across the studies which largely related to the content of material delivered and specific therapeutic techniques employed (see Table 2). Consistently reported modifications are summarised in Table 3. It is important to note that many interventions for anxiety focus on improving social skills (e.g. Storch et al. 2013; White et al. 2013; Wood et al. 2009a, b, 2015), but none of these studies report an improvement in child-reported anxiety and White et al. (2013) found no effect of the MASSI programme which specifically targets social skills and anxiety. As such, although this is a modification, it is not one that appears to be recommended for use in isolation.

\section{Obsessive-Compulsive Disorder}

One study was identified which met the eligibility criteria for the current study. Russell et al. (2013) recruited 46 participants aged 14-65 years from a range of clinical settings including ASD clinics, adult and paediatric OCD clinics and CAMHS clinics, generating a clinically representative sample. Although this study largely recruited adult participants, $20 \%$ of the sample were aged 18 or under and analysis revealed that outcomes were the same for adults and young people. All participants had a verbal IQ of $>70$, but specific ASD diagnosis was not described. ASD diagnosis was independently validated using the ADI-R and ADOS, and the presence of OCD was verified with the Yale-Brown Obsessive-Compulsive Scale (YBOCS). Participants were recruited and randomly allocated to the CBT or Anxiety Management (AM) treatment group indicating an appropriate selection of clinic-based controls. History of OCD was established in both groups, and baseline symptom severity was controlled for in the analysis, reducing risk of bias to detect effects.

The intervention included up to $20 \times 1$-h individual sessions, although there was great variation in this with treatment completers being defined as attending a minimum of seven sessions. The CBT intervention was based on a treatment manual designed specifically for clients with ASD and included four components of CBT: psychoeducation about anxiety and the cognitive cycle, problem solving, cognitive restructuring and Exposure and Response Prevention (ERP). The intervention was compared with an AM intervention providing psychoeducation about anxiety and relaxation strategies. The main outcome measure, the YBOCS, was administered by independent evaluators blind to treatment condition at pre-, post- and follow-up sessions. Dropout rate was comparable across groups reducing risk of bias in ascertainment of efficacy. Findings indicated a significant reduction in OCD symptoms and a greater number of treatment responders in the CBT compared to AM group, but differences were not significant between groups. Effect sizes were small which is again consistent with the lack of significant difference between groups. However, this study design achieved an overall NOS score of 8 indicating low risk of bias, implying that findings of a lack of significance of CBT over anxiety management for OCD in this population should be considered a reliable finding.

Modifications This study included five of the NICE recommended modifications. Parents were not included, but this would not have been appropriate given the broad range of ages of participants, and there was no report of offering regular breaks. Additional modifications employed were disorder specific (see Table 4) confirming the need to differentiate from anxiety treatment.

Table 4 Summary of additional modifications made to the treatment of OCD in an ASD population

Recommended adaptations to CBT for OCD

- Up to 20 sessions to allow for a longer assessment period to differentiate compulsions from rituals and access for meanings attributed to intrusive thoughts

- Standard treatment approach for OCD employed intervention predominantly focused on ERP using a graded hierarchy and home practices 


\section{Depression}

Only one study was identified which met the eligibility criteria for the current study for treating depression in young people with ASD. McGillivray and Evert (2014) recruited 32 high functioning participants (23 males) from a community sample aged between 15 and 25 years, diagnosed with Asperger's syndrome $(n=23)$ and HFA $(n=9)$, through community-based advertisements. ASD diagnosis was confirmed with a telephone interview only, and the presence of depression was determined through a self-report questionnaire (Depression Anxiety Stress Scales) indicating poor representativeness and validation of case definition. Participants were recruited and randomly allocated to CBT or WL generating an appropriate selection of clinic-based controls. History of depression was assessed and reported in both groups. There were no significant differences between groups on demographics, but comparability of cases and controls was not ensured through matching variables in design or controlling for differences/baseline symptoms in analysis.

This intervention was developed specifically for people with depression and ASD and was based on the literature reporting that social difficulties associated with ASD can lead to negative views of self and relationships with others. The study was a controlled trial with an intervention compared to WL group. The intervention was developed as a brief manualised programme named 'Think Well, Feel Well and Be Well' and comprised of $9 \times 2$-h group sessions. Four components of CBT were incorporated, namely psychoeducation, somatic management, problem solving and cognitive restructuring. The intervention had a cognitive rather than behavioural emphasis. Dropouts from either group were not described.

Participants from both groups completed the DASS and the Automatic Thoughts Questionnaire. There was no effect of intervention in terms of the between group comparison. However, those in the CBT group who scored above the clinical cutoff at baseline had significantly reduced depression symptoms when compared to WL post-treatment. There was no significant effect of intervention on negative automatic thoughts compared to WL, despite the substantial cognitive component. Effect sizes were small but $60 \%$ in the CBT group were reported to make substantial improvements compared to $20 \%$ in the WL and gains were maintained at 9month follow-up.

This study design was relatively flawed in terms of areas of potential bias and achieved an overall NOS score of 3 indicating high risk. Thus, the findings should be interpreted with caution, but this is the only study of its kind investigating the impact of a CBT intervention for young people with ASD and depression and should therefore be reviewed as a benchmark from which to develop more robust interventions.
Table 5 Summary of specific modifications to treat depression in YP with ASDs

Recommended adaptations to CBT for depression

- Shorter programme (9 weeks)

- Strong emphasis on challenging negative thoughts

- Introduction of thought records

- Mindfulness rather than relaxation

- Less of a behavioural emphasis consistent with cognitive not behavioural activation intervention

- Strategies to manage the 'internal critic' through thought catching and replacing

- Teaching links between behaviour and mood

- Improving social resources

Modifications This study included only one of the NICE recommended guidelines: emotional recognition training. As with the OCD intervention, some recommendations would not be applicable, such as including parents due to the age range of the sample. This study did employ a range of additional modifications which are disorder specific, again confirming the need to differentiate from anxiety treatment (Table 5).

\section{Discussion}

This review included 12 studies exploring the impact of CBT $\mathrm{v}$ WL, TAU or less structured intervention programmes in 501 young people with ASD and a comorbid mental health difficulty. In order to meet eligibility, studies had to report a significant effect of the intervention on mental health symptoms and meet rigorous design criteria. Studies recruited a mix of clinic and community-based samples, used group and individual therapeutic interventions based on modified or specially developed manuals containing most, if not all, components required for CBT. Measurement of outcome was typically multi-modal comprising child, parent and clinician reports. Results indicated a significant reduction in mental health symptoms as a result of the intervention on at least one outcome measure. With the exception of McGillivray and Evert's (2014) study, all interventions achieved moderate to low risk of bias indicating a high quality of study design and implying that conclusions can be drawn with relative confidence.

Largely consistent with the NICE guidelines (NICE CG170 2013), the evidence suggests that CBT with specific adaptations can be an effective intervention for young people with ASD and comorbid anxiety, OCD and possibly depression. The limited evidence comparing CBT to an active intervention has not shown CBT to be superior in reducing symptoms of anxiety (Sung et al. 2011) or OCD (Russell et al. 2013). However, CBT yields significant clinical improvements in this population, can be delivered in a group or 
individual format with equal success and in a relatively timelimited intervention. Furthermore, studies employing CBT interventions to target the core features of ASD, which may increase vulnerability to develop mental ill health, have been equally successful (e.g. impaired social skills (Wood et al. 2009b), emotion regulation (Scarpa and Reyes 2011), reduced independence of daily living (Drahota et al. 2011) and executive dysfunction (Kenworthy et al. 2014). This suggests that, among this population, modified CBT may reduce immediate emotional distress and act to enhance resilience against developing future mood or affective disorders. More research is needed but modified 'cognitive methods appear to be a feasible treatment option when applied to the mental health needs of such young people' (Donoghue et al. 2011, p. 98).

In consideration of the primary research questions, among these studies, the extent to which modifications recommended by NICE are employed varied greatly from one (McGillivray and Evert 2014) to all seven (Reaven et al. 2009, 2012a). The adaptations identified follow recommendations for enhancing accessibility of CBT for young people with ASD by making sessions more concrete, practical and creative with a general emphasis on affect recognition, increased exposure opportunities and parental involvement (e.g. Donoghue et al. 2011; Rotheram-Fuller and MacMullen 2011; White et al. 2009). However, the broad variation across studies may suggest that just employing basic modifications to delivery is not sufficient to meet the needs of young people with ASD.

The findings of the current review imply that the NICE guidelines may be a useful template from which to begin adapting interventions but additional modifications are also being routinely employed within research trials to meet neurodevelopmental needs and successfully treat the symptoms of comorbid mental health disorders. Additional modifications identified within studies include (i) add-on components for parents rather than just involving them in the child intervention (e.g. Reaven et al. 2009, 2012a) and (ii) techniques such as social stories, acronyms and role plays to accommodate features of ASD including literal understanding and theory of mind deficits (e.g. Kenworthy et al. 2014; Rotheram-Fuller and MacMullen 2011; Wood et al. 2014). The successful results reported in the studies reviewed are highly promising but caution must be taken when attributing successes to the modifications specifically. To date, there are no published studies comparing modified CBT to standard CBT interventions for this population. It is possible that the active component yielding positive results is the CBT rather than the modifications. However, the Social Recreation (Sung et al. 2011) and Anxiety Management (Russell et al. 2013) programmes which were comparator interventions modified for the needs of people with ASD were as effective as the CBT intervention, suggesting that such modifications may be essential, active treatment ingredients.

Despite the dearth of literature exploring interventions for disorders other than anxiety, there is a trend to suggest that modifications to CBT should be disorder specific, as they would be for a typically developing population. Research would seem to suggest that the underlying cognitive mechanisms and manifestation of OCD, depression and even PTSD are the same in typically developing young people and those with ASD (e.g. Barnhill and Smith Myles 2001; Boyd et al. 2012; Cook et al. 1993; Ghaziuddin et al. 2002; Hedley and Young 2006; Howlin and Clemments 1995; Mehtar and Mukaddes 2011; Whitehouse et al. 2009). This suggests that interventions should be tailored to directly target these symptoms and/or disorder-specific manuals should be adapted to treat each separate disorder. Such findings have also led to consideration that 'development of a cognitive model specific to this population is necessary in guiding therapeutic interventions' (Ozsivadjian and Knott 2011, p. 212).

There is some evidence to support the value of developing disorder-specific CBT manuals for young people with ASD. For example, Russell et al. (2013) focused a large portion of their intervention on OCD-specific ERP, while McGillivray and Evert (2014) employed techniques such as mindfulness and dysfunctional thought records from the typically developing literature and both studies resulted in reduced symptomatology. Similarly, well-cited case studies describe modifying and implementing an OCD-specific treatment manual (March and Mulle 1998) and achieving symptom remission and recovery (e.g. Lehmkuhl et al. 2008; Reaven and Hepburn 2003). There is clearly a need for replication studies in each of these areas, but findings tentatively point to the benefit of developing tailored interventions which specifically meet the neurodevelopmental and mental health needs of this population.

\section{Clinical Implications}

Collectively, the findings of this review highlight several key practice points for clinicians. Namely, CBT should be offered as an intervention for young people with ASD and comorbid mental health problems including anxiety disorders, OCD and depression. Modifications should be applied to both the content and the structure of manualised interventions.

An enhanced emotional recognition component should be included to support young people to develop a way to communicate their feelings and recognise change in intensity of emotion throughout therapy. Cognitive components should be retained but may need to include less of a collaborative or Socratic approach and employ more structure, forced choice 
or multiple choice, with concrete, rather than hypothetical, examples. Sessions should employ clear, concrete visual prompts which act to enhance verbally mediated material and reduce the intensity of a 1:1 therapy session. Special interests can be used to provide engaging, concrete analogies to illustrate therapeutic principles, ensuring a thorough understanding of the model underpinning the intervention. Interventions should try and include parents within sessions where possible to support in-session communication and between session tasks. This can be enhanced by therapist modelling of therapeutic techniques, such as ERP, within sessions in the presence of parents.

Session materials should also be tailored to be age appropriate. The majority of studies reviewed included children, but Reaven et al. (2012b) distinguish the developmental needs of adolescents from children and developed the Facing your Fears Adolescent programme to meet those needs accordingly. For example, the parent component focuses on features of the parent-teen relationship relevant to navigating the transition through adolescence, and iPads are used to convey concepts of therapy and encourage home practice in a way that is accessible to typical adolescent functioning.

As indicated, findings tentatively point to the need to include disorder-specific modifications to intervention. The review identified only two studies evaluating the impact of disorder-specific protocols, for depression and OCD. Methodological weaknesses however limit the extent to which the results of these studies can reliably inform future practice. Elevated prevalence rates of mental health problems such as OCD, PTSD and depression in young people with ASD (e.g. Ghaziuddin et al. 1998; Leyfer et al. 2006; Mehtar and Mukaddes 2011) suggest that robust evaluations of disorder-specific treatment protocols are warranted.

Finally, all studies included in this review recruited participants with average or above IQ making it difficult to know how generalizable modified CBT is for young people with impaired language skills or more pervasive developmental delay often associated with autism (Lang et al. 2010; Reaven 2011; Van Steensel et al. 2011; Wood et al. 2011). It is beyond the scope of this review to make recommendations for young people with autism and significant impairments in language or intellectual disability (ID). The practice implications may not specific to ASD or might need to be combined with practice recommendations for people with ID. For example, Hassiotis et al. (2012) have published a therapist manual for adapting CBT for people with ID, and there is considerable overlap with some of the recommendations from this review. The authors point to the need to use visual prompts, include carers and take a disorderspecific approach, suggesting that such modifications may have a universally beneficial impact for this population but further research is required.

\section{Limitations}

This review employs rigorous criteria to identify effective studies investigating CBT for a range of mental health problems. However, there are several methodological aspects which introduce the potential for bias. The lack of two independent raters for study selection and data extraction may be a potential source of bias. All authors have qualifications and experience in evaluating research, and many checks were put in place in an effort to limit bias, including the second author supervising each stage of the process, the use of the NOS to rate studies and multiple revisions to ensure an accurate narrative of findings. The bias introduced through having a single rater, however, should not be overlooked.

This review relied on published studies. Published work may be more likely to report larger effect sizes than unpublished studies (Hopewell et al. 2007; Reichow et al. 2011). However, bias can also be introduced by reporting effects of unpublished trials which have not been peer-reviewed for methodological rigour and may not be representative of all unpublished data (Egger et al. 2003). As such, the decision was taken to review studies which had passed the peer review process.

The inclusion criteria also specified using studies published in English. During the search, no non-English studies were identified but the possibility of a missed area of research should be acknowledged. Finally, only studies with a comparator group and significant effect of treatment were included. This may have meant effective modifications from nonsignificant treatment studies were omitted, or ineffective modifications from studies with a significant treatment effect were mis-identified.

\section{Conclusion}

Limitations notwithstanding, the current study adds to the understanding of what works for young people with ASD attending mental health services for psychological interventions. Findings are clinically relevant and synthesise results from the most robust published studies in the area. This review identifies meaningful techniques and methods of delivery which can support young people with ASD to engage with a programme of therapy and experience reduction in anxiety. Preliminary evidence also points to the efficacy of targeted CBT for OCD and depression. There remains a need for future research, but in the absence of such work, standardised treatment manuals for typically developing young people may 
effectively alleviate mental health symptoms in young people with ASD when adapted with NICE recommended modifications to structure and disorder-specific modifications to content.

Open Access This article is distributed under the terms of the Creative Commons Attribution 4.0 International License (http:// creativecommons.org/licenses/by/4.0/), which permits unrestricted use, distribution, and reproduction in any medium, provided you give appropriate credit to the original author(s) and the source, provide a link to the Creative Commons license, and indicate if changes were made.

\section{References}

Attwood, T. (2002). James and the Maths test. Unpublished Manuscript. Attwood, T. (2004). Exploring feelings: cognitive behaviour therapy to manage anxiety. Arlington: Future Horizons.

Baird, G., Kendall, T., Bovell, V., Buckley, C., Charman, T., Gould, N., Whitlingum, G. (2013). Autism: the management and support of children and young people on the autistic spectrum: NICE guideline draft: National Institute for Health and Care Excellence.

Barnhill, G. P., \& Smith Myles, B. (2001). Attributional style and depression in adolescents with Asperger syndrome. Journal of Positive Behaviour Interventions, 3, 175-182.

Baron-Cohen, S., Leslie, A. M., \& Frith, U. (1985). Does the autistic child have a theory of mind? Cognition, 21, 37-46.

Baron-Cohen, S., Wheelwright, S., Skinner, R., Martin, J., \& Clubley, E. (2001). The autism-spectrum quotient (AQ): evidence from Asperger syndrome/high-functioning autism, males and females, scientists and mathematicians. Journal of Autism and Developmental Disorders, 31, 5-17.

Boyd, B. A., McDonough, S. G., \& Bodfish, J. W. (2012). Evidencebased behavioural interventions for repetitive behaviours in autism. Journal of Autism and Developmental Disorders, 42, 1236-1248.

Cartwright-Hatton, S., Roberts, C., Chitsabesan, P., Fothergill, C., \& Harrington, R. (2004). Systematic review of the efficacy of cognitive behaviour therapies for childhood and adolescent anxiety disorders. British Journal of Clinical Psychology, 43, 421-436.

Chalfant, A. M., Rapee, R., \& Carroll, L. (2007). Treating anxiety disorders in children with high functioning autism spectrum disorders: a controlled trial. Journal of Autism and Developmental Disorders, $37,1842-1857$.

Cook, E. H., Kieffer, J. E., Charak, D. A., \& Leventhal, B. L. (1993). Autistic disorder and post-traumatic stress disorder. Journal of the American Academy of Child and Adolescent Psychiatry, 32, 12921294.

Donoghue, K., Stallard, P., \& Kucia, J. (2011). The clinical practice of cognitive behavioural therapy for children and young people with a diagnosis of Asperger's syndrome. Clinical Child Psychology and Psychiatry, 16, 89-102.

Drahota, A., Wood, J. J., Sze, K., \& Van Dyke, M. (2011). Effects of cognitive behavioural therapy on daily living skills in children with high-functioning autism and concurrent anxiety disorders. Journal of Autism and Developmental Disorders, 41, 257-265.

Egger, M., Juni, P., Bartlett, C., Holenstein, F., \& Sterne, J. (2003). How important are comprehensive literature searches and the assessment of trial quality in systematic reviews? Empirical study. Health Technology Assessment, 7, 1-76.

Fuji, C., Renno, P., McLeod, B. D., Lin, C. E., Decker, K., Zielinski, K., \& Wood, J. J. (2013). Intensive cognitive behavioural therapy for anxiety disorders in school-aged children with autism: a preliminary comparison with treatment-as-usual. School Mental Health, 5, 2537.

Ghaziuddin, M., Weidmer-Mikhail, E., \& Ghaziuddin, M. (1998). Comorbidity of Asperger syndrome: a preliminary report. Journal of Intellectual Disability Research, 42, 279-283.

Ghaziuddin, M., Ghaziuddin, N., \& Greden, J. (2002). Depression in persons with autism: implications for research and clinical care. Journal of Autism and Developmental Disorders, 32, 299-306.

Gillberg, C., \& Billstedt, E. (2000). Autism and Asperger syndrome: coexistence with other clinical disorders. Acta Psychiatrica Scandinavica, 102, 321-330.

Hassiotis, A., Serfaty, M., Azam, K., Martin, S., Strydom, A., \& King, M. (2012). A manual of cognitive behaviour therapy for people with learning disabilities and common mental disorders. London: Camden \& Islington NHS Foundation Trust and University College London.

Hedley, D., \& Young, R. (2006). Social comparison processes and depressive symptoms in children and adolescents with Asperger syndrome. Autism, 10, 139-153.

Hopewell, S., Clarke, M., Stewart, L., \& Tierney, J. (2007). Time to publication for results of clinical trials. Cochrane Database of Systematic Reviews. doi:10.1002/14651858.MR000011.pub2.

Howlin, P., \& Clemments, J. (1995). Is it possible to assess the impact of abuse on children with pervasive developmental disorders. Journal of Autism and Developmental Disorders, 25, 337-354.

Kannabiran, M., \& McCarthy, J. (2009). The mental health needs of people with autism spectrum disorders. Psychiatry, 8, 398-401.

Kendall, P. C. (1992). Coping cat workbook. Ardmore: Workbook Publishing.

Kenworthy, L., Anthony, L., Naiman, D. Q., Cannon, L., Wills, M. C., Luong-Tran, C., \& Wallace, G. L. (2014). Randomized controlled effectiveness trial of executive function intervention for children on the autism spectrum. The Journal of Child Psychology and Psychiatry, 55, 374-383.

King, R., \& Desaulnier, C. L. (2011). Commentary: complex posttraumatic stress disorder. Implications for individuals with autism spectrum disorders - part II. Journal on Developmental Disabilities, $17,47-59$.

Lang, R., Regester, A., Lauderdale, S., Kristen, A., \& Haring, A. (2010). Treatment of anxiety in autism spectrum disorders using cognitive behaviour therapy: a systematic review. Developmental Neurorehabilitation, 13, 53-63.

Langdon, P. E., Murphy, G. H., Wilson, E., Shepstone, L., Fowler, D., Heavens, D., \& Russell, A. (2013). Asperger syndrome and anxiety disorders (PAsSA) treatment trial: a study protocol of a pilot multicentre single-blind, randomised crossover trial of group cognitive behaviour therapy. BMJ Open, 3, 1-6.

Lehmkuhl, H. D., Storch, E. A., Bodfish, J. W., \& Geffken, G. R. (2008). Brief report: exposure and response prevention for obsessive compulsive disorder in a 12-year-old with autism. Journal of Autism and Developmental Disorders, 38, 977-981.

Leyfer, O. T., Folstein, S. E., Bacalman, S., Davis, N. O., Dinh, E., Morgan, J., \& Lainhart, J. E. (2006). Comorbid psychiatric disorders in children with autism: interview development and rates of disorders. Journal of Autism and Developmental Disorders, 36, 849-861.

Liberati, A., Altman, D. G., Tetzlaff, J., Mulrow, C., Gotzsche, P. C., Ioannidis, J. P. A., \& Moher, D. (2009). The PRISMA statement for reporting systematic reviews and meta-analyses of studies that evaluate healthcare interventions: explanation and elaboration. $B M J$ Online, 339, b2700. doi:10.1136/bmj.b2700.

Lickel, A., Maclean, W. E., Blakeley-Smith, A., \& Hepburn, S. (2012). Assessment of the prerequisite skills for cognitive behavioural therapy in children with and without autism spectrum disorders. Journal of Developmental Disorders, 42, 992-1000. 
Lord, C., Rutter, M., Goode, S., Heemsbergen, J., Jordan, H., Mawhood, L., \& Schopler, E.(1989). Autism diagnostic observation schedule: a standardized observation of communicative and social behavior. Journal of Autism and Developmental Disorders, 19, 185-212.

Lyneham, H. J., Abbott, M. J., Wignall, A., \& Rapee, R. (2003). The cool kids family program-therapist manual. Sydney: Macquarie University.

March, J. S., \& Mulle, K. (1998). OCD in children and adolescents: a cognitive-behavioural treatment manual. New York: Guilford Press.

Matson, J. L., \& Nebel-Schwalm, M. S. (2007). Comorbid psychopathology with autism spectrum disorder in children: an overview. Research in Developmental Disabilities, 28, 341-352.

McGillivray, J. A., \& Evert, H. T. (2014). Group cognitive behavioural therapy program shows potential in reducing symptoms of depression and stress among young people with ASD. Journal of Autism and Developmental Disorders, 44, 2041-51.

McNally Keehn, R. H., Lincoln, A. J., Brown, M. Z., \& Chavira, D. A. (2013). The coping cat program for children with anxiety and autism spectrum disorder: a pilot randomized controlled trial. Journal of Autism and Developmental Disorders, 43, 57-67.

Mehtar, M., \& Mukaddes, N. M. (2011). Posttraumatic stress disorders in individuals with a diagnosis of autism spectrum disorders. Research in Autism Spectrum Disorders, 5, 539-546.

Minshew, N. J., Goldstein, G., \& Siegel, D. J. (1997). Neuropsychological functioning in autism: profile of a complex information processing disorder. Journal of International Neuropsychological Society, 3, 303-316.

Moher, D., Liberati, A., Tetzlaff, J., Altman, D. G., \& The PRISMA Group. (2009). Preferred reporting items for systematic reviews and meta-analyses: the PRISMA statement. PLoS Med, 6(6), e1000097. doi:10.1371/journal.pmed1000097.

Nadeau, J. M., Arnold, E. B., Storch, E. A., \& Lewin, A. B. (2014). Family cognitive-behavioural treatment for a child with autism and comorbid obsessive compulsive disorder. Clinical Case Studies, 13, $22-36$.

NICE CG170. (2013). Autism: the management and support of children and young people on the autism spectrum. National Institute for Care and Health Excellence.

Nuata, M.H., Scholing, A., Rapee, R.M., Abbott, M., Spence, S.H., \& Waters, A. (2004). A parent-report measure of children's anxiety: Psychometric properties and comparison with child-report in a clinic and normal sample. Behaviour Research and Therapy, 42, 813-839.

Ooi, Y. P., Lam, C. M., Sung, M., Tan, W. T. S., Goh, T. J., Fung, D. S. S., \& Chua, A. (2008). Effects of cognitive-behavioural therapy on anxiety for children with high-functioning autistic spectrum disorders. Singapore Medical Journal, 49, 215-220.

Ozonoff, S., Pennington, B. F., \& Rodgers, S. J. (1991). Executive functioning deficits in high-functioning autistic individuals: relationship to theory of mind. Journal of Child Psychology and Psychiatry and Applied Disciplines, 32, 1081-1105.

Ozsivadjian, A., \& Knott, F. (2011). Anxiety problems in young people with autism spectrum disorder: a case series. Clinical Child Psychology and Psychiatry, 16, 203-214.

POTS. (2004). Cognitive behaviour therapy, sertraline and their combination for children and adolescents with obsessive-compulsive disorder. Journal of American Medical Association, 27, 1969-1976.

Reaven, J. (2011). The treatment of anxiety symptoms in youth with high functioning autism spectrum disorders: developmental considerations for parents. Brain Research, 1380, 255-263.

Reaven, J., \& Hepburn, S. (2003). Cognitive-behavioural treatment of obsessive-compulsive disorder in a child with Asperger syndrome. Autism, 7, 145-164.

Reaven, J., Blakeley-Smith, A., Nichols, S., Dasari, M., Flanigan, E., \& Hepburn, S. (2009). Cognitive-behavioural group treatment for anxiety symptoms in children with high-functioning autism spectrum disorders: a pilot study. Focus on Autism and Other Developmental Disabilities, 24, 27-37.

Reaven, J., Blakeley-Smith, A., Culhane-Shelburne, K., \& Hepburn, S. (2012a). Group cognitive behaviour therapy for children with high functioning autism spectrum disorders and anxiety: a randomized trial. The Journal of Child Psychology and Psychiatry, 53, 410-419.

Reaven, J., Blakeley-Smith, A., Leuthe, E., Moody, E., \& Hepburn, S. (2012b). Facing your fears in adolescence: cognitive-behavioural therapy for high functioning autism spectrum disorders and anxiety. Autism Research and Treatment, 2012, 1-13.

Reichow, B., Doehring, P., Ciccetti, D. V., \& Volkmar, F. R. (2011). Evidence-based practices and treatments for children with autism. New York: Springer.

Reinecke, M. A., Ryan, N. E., \& Dubois, D. L. (1998). Cognitivebehavioural therapy of depression and depressive symptoms during adolescence: a review and meta-analysis. Journal of the American Academy of Child and Adolescent Psychiatry, 37, 26-34.

Rotheram-Fuller, E., \& MacMullen, L. (2011). Cognitive behavioural therapy for children with autism spectrum disorders. Psychology in the Schools, 48, 263-271.

Russell, A. J., Jassi, A., Fullana, M. A., Mack, H., Johnston, K., Heyman, I., \& Mataix-Cols, D. (2013). Cognitive behaviour therapy for comorbid obsessive-compulsive disorder in high-functioning autism spectrum disorders: a randomised controlled trial. Depression and Anxiety, 30, 679-708.

Scarpa, A., \& Reyes, N. M. (2011). Improving emotion regulation with CBT in young children with high functioning autism spectrum disorders: a pilot study. Behavioural and Cognitive Psychotherapy, 39, 495-500.

Scattone, D., \& Mong, M. (2013). Cognitive behaviour therapy in the treatment of anxiety for adolescents and adults with autism spectrum disorders. Psychology in the Schools, 50, 923-935.

Schleismann, K. D., \& Gillis, J. M. (2011). The treatment of social phobia in a young boy with Asperger's disorder. Cognitive and Behavioural Practice, 18, 515-529.

Silverman, W.K., \& Albano, A.M. (1996). The Anxiety Disorders Interview Schedule for DSM-IV - Child and Parent Versions. Psychological Corporation, San Antonio, TX

Simonoff, E., Pickles, A., Charman, T., Chandler, S., Loucas, T., \& Baird, G. (2008). Psychiatric disorders in children with autism spectrum disorders: prevalence, comorbidity and associated factors in a population-derived sample. Journal of American Child and Adolescent Psychiatry, 47, 921-929.

Sofronoff, K., Attwood, T., \& Hinton, S. (2005). A randomized controlled trial of a CBT intervention for anxiety in children with Asperger syndrome. Journal of Child Psychology and Psychiatry, 46, 1152-1160.

Storch, E. A., Arnold, E. B., Lewin, A. B., Nadeau, J. M., Jones, A. M., De Nadai, A. S., \& Murphy, T. K. (2013). The effect of cognitivebehavioural therapy versus treatment as usual for anxiety in children with autism spectrum disorders: a randomized controlled trial. Journal of the American Academy of Child and Adolescent Psychiatry, 52, 132-142.

Sung, M., Ooi, Y. P., Goh, T. J., Pathy, P., Fung, D. S. S., Ang, R. P., \& Lam, C. M. (2011). Effects of cognitive-behavioural therapy on anxiety in children with autism spectrum disorders: a randomized controlled trial. Child Psychiatry and Human Development, 42, 634-649.

Sze, K., \& Wood, J. J. (2007). Cognitive behavioural treatment of comorbid anxiety disorders and social difficulties in children with highfunctioning autism: a case report. Journal of Contemporary Psychotherapy, 37, 133-143.

Sze, K., \& Wood, J. J. (2008). Enhancing CBT for the treatment of autism spectrum disorders and concurrent anxiety. Behavioural and Cognitive Psychotherapy, 36, 403-409. 
Van Steensel, F. J. A., Bogels, S. M., \& Perrin, S. (2011). Anxiety disorders in children and adolescents with autistic spectrum disorders: a meta-analysis. Clinical Child and Family Psychology Review, 14, 302-317.

Vasa, R. A., Carroll, L. M., Nozzolillo, A. A., Mahajan, R., Mazurek, M. O., Bennett, A. E., Wink, L. K., \& Bernal, M. P. (2014). A systematic review of treatments for anxiety in youth with autism spectrum disorders. Journal of Autism and Developmental Disorders, 44, 3215-3229.

Velting, O. N., Setzer, N. J., \& Albano, A. M. (2004). Update on advances in assessment and cognitive behavioural treatment of anxiety disorders in children and adolescents. Professional Psychology: Research and Practice, 35, 42-54.

Wethington, H. R., Hahn, R., Fuqua-Whitley, D. S., Sipe, T. A., Crosby, A. E., Johnson, R. L., \& Services, T. f. o. c. p. (2008). The effectiveness of interventions to reduce psychological harm from traumatic events among children and adolescents. American Journal of Preventative Medicine, 35, 287-313.

White, S. W., Ollendick, T., Scahill, L., Oswald, D., \& Albano, A. M. (2009). Preliminary efficacy of a cognitive-behavioural treatment program for anxious youth with autism spectrum disorders. Journal of Autism and Developmental Disorders, 39, 1652-1662.

White, S. W., Albano, A. M., Johnson, C. R., Kasari, C., Ollendick, T., Klin, A., Scahill, L. (2010). Development of a cognitive-behavioural intervention program to treat anxiety and social deficits in teens with high-functioning autism. Clinical Child and Family Psychology Review, 13, 77-90.

White, S. W., Ollendick, T., Albano, A. M., Oswald, D., Johnson, C., Southam-Gerow, M. A., Kim, I., \& Scahill, L. (2013). Randomised controlled trial: multimodal anxiety and social skill intervention for adolescents with autism spectrum disorder. Journal of Autism and Developmental Disorders, 43, 382-394.
Whitehouse, A. J. O., Durkin, K., Jaquet, E., \& Ziatas, K. (2009). Friendship, loneliness and depression in adolescents with Asperger's syndrome. Journal of Adolescence, 32, 309-322.

Wistow, R., \& Barnes, D. (2009). A profile of child and adolescent mental health services in England 2007/8. Durham: Durham University, School of Applied Social Sciences.

Wood, J. J., \& Drahota, A. (2005). Behavioural interventions for anxiety in children with autism. Los Angeles: University of California.

Wood, J. J., \& McLeod, B. D. (2008). Child anxiety disorders: a treatment manual for practitioners. New York: Norton.

Wood, J. J., Drahota, A., Sze, K., Har, K., Chui, A., \& Langer, D. (2009a). Cognitive behavioural therapy for anxiety in children with autism spectrum disorders: a randomized controlled trial. The Journal of Child Psychology and Psychiatry, 50, 224-234.

Wood, J. J., Drahota, A., Sze, K., Van Dyke, M., Decker, K., Fuji, C., \& Spiker, M. (2009b). Brief report: effects of cognitive behavioural therapy on parent-reported autism symptoms in school-aged children with high-functioning autism. Journal of Autism and Developmental Disorders, 39, 1608-1612.

Wood, J. J., Fuji, C., \& Renno, P. (2011). Cognitive behavioural therapy in high functioning autism: review and recommendations for treatment development. In B. Reichow, P. Doehring, D. V. Ciccetti, \& F. R. Volkmar (Eds.), Evidence-based practices and treatments for children with autism (pp. 197-231). New York: Springer.

Wood, J. J., Fuji, C., Renno, P., \& Van Dyke, M. (2014). Impact of cognitive behavioural therapy on observed autism symptom severity during school recess: a preliminary randomized controlled trial. Journal of Autism and Developmental Disorders, 44, 2264-76.

Wood, J. J., Ehrenreich-May, J., Alessandri, M., Fuji, C., Renno, P., Laugeson, E., \& Storch, E. A. (2015). Cognitive behavioural therapy for early adolescents with autism spectrum disorders and clinical anxiety: a randomized controlled trial. Behaviour Therapy, 46, 7-19. 